\title{
Dielectric constant of flagellin proteins measured by scanning dielectric microscopy
}

DOI:

10.1039/c8nr06190d

Document Version

Accepted author manuscript

Link to publication record in Manchester Research Explorer

\section{Citation for published version (APA):}

Lozano, H., Fabregas, R., Blanco-Cabra, N., Millan-Solsona, R., Torrents, E., Fumagalli, L., \& Gomila, G. (2018). Dielectric constant of flagellin proteins measured by scanning dielectric microscopy. Nanoscale, 10(40), 1918819194. https://doi.org/10.1039/c8nr06190d

\section{Published in:}

Nanoscale

\section{Citing this paper}

Please note that where the full-text provided on Manchester Research Explorer is the Author Accepted Manuscript or Proof version this may differ from the final Published version. If citing, it is advised that you check and use the publisher's definitive version.

\section{General rights}

Copyright and moral rights for the publications made accessible in the Research Explorer are retained by the authors and/or other copyright owners and it is a condition of accessing publications that users recognise and abide by the legal requirements associated with these rights.

\section{Takedown policy}

If you believe that this document breaches copyright please refer to the University of Manchester's Takedown Procedures [http://man.ac.uk/04Y6Bo] or contact uml.scholarlycommunications@manchester.ac.uk providing relevant details, so we can investigate your claim.

\section{OPEN ACCESS}




\section{Nanoscale}

\section{ARTICLE}

\section{Dielectric constant of flagellin proteins measured by scanning dielectric microscopy}

Received 00th January 20xx, Accepted 00th January 20xx

DOI: $10.1039 / x 0 x \times 00000 x$

www.rsc.org/

\author{
Helena Lozano ${ }^{a, \ddagger}$, Rene Fabregas ${ }^{a, \ddagger}$, Nuria Blanco-Cabra ${ }^{b}$, Rubén Millán-Solsona ${ }^{a, c}$, Eduard To- \\ rrents $^{b}$, Laura Fumagallid,e, Gabriel Gomila*,a,c
}

\begin{abstract}
The dielectric constant of flagellin proteins in flagellar bacterial filaments $\sim 10-20 \mathrm{~nm}$ in diameter is measured using Scanning Dielectric Microscopy. We obtain for two different bacterial species (Shewanella oneidensis MR-1 and Pseudo-monas aeruginosa PAO1) similar relative dielectric constant values $\varepsilon_{S_{0}}=4.3 \pm 0.6$ and $\varepsilon_{P_{a}}=4.5 \pm 0.7$, respectively, despite their different structure and aminoacid sequence. Present results show the applicability of Scanning Dielectric Microscopy to nanoscale filamentous protein complexes, and to general 3D macromolecular protein geometries, thus opening new avenues to study the relationship between dielectric response and protein structure and function.
\end{abstract}

\section{Introduction}

The electric polarizability of proteins, represented in mean field theories by the relative dielectric constant (1), (2), has long been recognized as a key parameter in determining the proteins structure and their electrostatic interactions with charged biomolecules and ligands (3), (4), (5), (6). The dielectric constant determines the degree by which electric fields are screened by the protein microscopic electric dipoles themselves. As such, it has a strong effect in charge-charge and charge-dipole interactions in, and between, proteins, and hence, in the electro-static energy contribution to protein folding, proteinprotein, protein-DNA and protein-charged ligand interactions (7).

Recently, we have developed a technique that we refer here as Scanning Dielectric Microscopy able to access directly the dielectric constant of small scale macromolecular biological systems (8), (9). The method has already enabled determining the dielectric constant of proteins in empty virus capsids and in

a. Nanoscale Bioelectrical Characterization, Institute for Bioengineering of Catalonia (IBEC), The Barcelona Institute of Science and Technology (BIST), c/ Baldiri i Reixac 11-15, 08028, Barcelona, Spain

b. Bacterial Infections: Antimicrobial Therapies, Institute for Bioengineering of Catalonia (IBEC), The Barcelona Institute of Science and Technology (BIST), c/ Baldiri i Reixac 11-15, 08028, Barcelona, Spain

Departament d'Enginyeria Electrònica i Biomèdica, Universitat de Barcelona, C/ Martí i Franqués 1, 08028, Barcelona, Spain

d. School of Physics and Astronomy, University of Manchester, Manchester M13 gPL, UK.

e. National Graphene Institute, University of Manchester, Manchester M13 9PL, UK.

${ }^{\ddagger}$ Equally contributing authors.

*Corresponding author: ggomila@ibecbarcelona.eu

Electronic Supplementary Information (ESI) available: Additional information is provided on tip deconvolution, tip calibration and tip-sample distance, dependence of the capacitance gradient on flagellum length, tip radius, relative dielectric constant, flagellum curvature, dielectric constant extraction from dielectric approach curves and validation with full EFM dielectric profiles, parameters for the different flagella analyzed, and numerical calculations. See DOI: $10.1039 / x 0 x \times 00000 x$ virus tails (8), (9), as well as, of proteins in sub-micrometric protein mono-layer patches (10). In all cases, the obtained protein relative dielectric constants in dry air conditions were $\varepsilon_{r}$ $\sim 3-4$, in good agreement with the values reported from bulk measurements on dry protein powders, $\varepsilon_{r} \sim 2-5$ (11), (12) and with theoretical predictions excluding charged side chains, $\varepsilon_{r} \sim 4$ (4).

In the present work, we extend the applicability of Scanning Dielectric Microscopy to measure the dielectric constant of macromolecular protein complexes forming sub-10 nm diameter nanoscale filamentous structures (and in general, to any sub-10 $\mathrm{nm}$ size 3D geometry). The present study, by dealing with low polarizable sub-10 $\mathrm{nm}$ nanofilaments, goes well beyond earlier studies on the conducting (rather than dielectric) properties of organic and inorganic nanofilament structures, such as, DNA (13), carbon nanotubes (13), (14), (15), or semiconductor nanowires (16). Filamentous macromolecular protein complexes include important protein systems like actin or myosin filaments, microtubules, intermediate filaments, amyloid fibers or bacterial polar flagella, which is the specific case considered in the present work. These systems are fundamental in the biology of cells and, in some cases, play a central role in important diseases (17).

\section{Results and discussion}

We measured the dielectric constant of the flagellin proteins in bacterial polar flagella of two different bacterial species, namely, Shewanella oneidensis MR-1 and Pseudomonas aeruginosa PAO1. Flagella are long-thin ( $10-20 \mathrm{~nm}$ diameter) whip like appendages that bacteria use to move towards the nutrients. They are composed of 11 flagellin protein monomers twisting every $\sim 5 \mathrm{~nm}$ (18). To measure the dielectric constant, we used Scanning Dielectric Microscopy (SDM) as introduced in Ref. (8) (see Materials and Methods section). Briefly, SDM 
combines Electrostatic Force Microscopy (EFM) measurements (19), (20), and finite element numerical calculations made with realistic geometrical models. Here, given the filamentous nature of the polar flagella we needed to use 3D electrostatic models. The dielectric constant measured in this way corresponds to a region of the flagellum containing a few hundred of flagellin protein monomers, as illustrated in the example of the calculated electric potential distribution shown in Fig. 1 (see also discussion below).

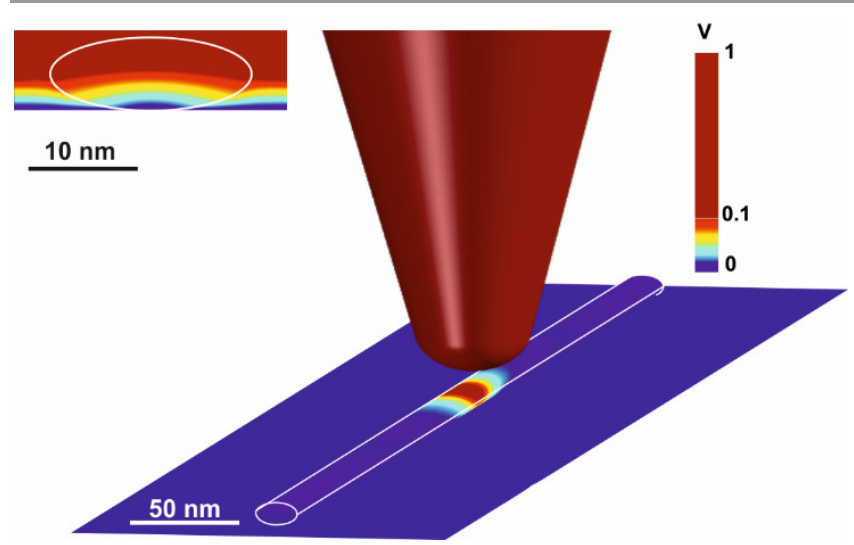

Figure 1: Example of the electric potential distribution obtained by solving the 3D tip flagellum electrostatic model by means of finite element numerical calculations. The simulations show that only a narrow portion of the flagellum, containing a few hundred of flagellin protein monomers, is probed. Parameters used in the numerical calculations: cone height $H=12.5 \mu \mathrm{m}$, cantilever width $W_{c}=3000 \mathrm{~nm}$ and length $L_{c}=3000 \mathrm{~nm}$, half cone angle $\vartheta=11.5^{\circ}$, apex radius $R=24 \mathrm{~nm}$, tip-substrate distance $z_{C H}=28 \mathrm{~nm}$, applied voltage $V=1 \mathrm{~V}$, flagellum height $h=8 \mathrm{~nm}$, width $w=18 \mathrm{~nm}$, length $I=1 \mu \mathrm{m}$, and relative dielectric constant, $\varepsilon_{r}=4$. Inset: electric potential distribution of a cross-section cut of the axial plane of the flagellum.

Figure 2a shows topographic atomic force microscopy (AFM) images of two $S$. oneidensis MR-1 and one $P$. aeruginosa PAO1 bacterial cells on a Highly Oriented Pyrolithic Graphite (HOPG) substrate, which show the presence of the polar flagella. Figures $2 \mathrm{~b}$ and $2 \mathrm{c}$ show zoom-ins around the respective flagella, while Fig. $2 \mathrm{~d}$ show the topographic cross-section profiles taken along the dashed lines in Figs. $2 \mathrm{~b}$ and $2 \mathrm{c}$. The dimensions of the $S$. oneindensis flagellum obtained from the topographic images after tip deconvolution (see Materials and Methods and Supplementary Information) are: height $h_{S o}=8.8 \pm 0.5 \mathrm{~nm}$, width $w_{s o}=18 \pm 2 \mathrm{~nm}$, and length $I_{s o}>1 \mu \mathrm{m}$. For the $P$. aeruginosa flagellum, we obtain $h_{P a}=12.8 \pm 0.5 \mathrm{~nm}, w_{P a}=27 \pm$ $2 \mathrm{~nm}$ and $I_{P a}>1 \mu \mathrm{m}$, where the error is the standard deviation over five profiles. The measured flagella dimensions are in good agreement with existing experimental data. For instance, Ref. (18) reports a near-atomic resolution cryo-Transmission Electron Microscopy (cryo-TEM) structure of flagellar filaments for different bacteria species, giving for $P$. aeruginosa a radius $\sim 8.5 \mathrm{~nm}$. With the AFM we have obtained a half height of $\sim 6.5$ $\mathrm{nm}$ and a half width of $\sim 13.5 \mathrm{~nm}$, giving an equivalent circular radius of $\sim 9.5 \mathrm{~nm}$, in excellent agreement with the cryo-TEM measurements. This result indicates that the drying and adsorption process only slightly alters the structure of the flagella, producing a slight flattening at nearly constant area. Figures $2 \mathrm{e}$ and $2 \mathrm{~g}$ show two sets of four constant height EFM capacitance gradient images obtained at distances $\left(z_{\mathrm{CH}}\right)$ ranging from $\sim 20 \mathrm{~nm}$ to $\sim 80 \mathrm{~nm}$ for the two types of bacterial flagella considered, respectively. The corresponding capacitance gradient cross-section profiles along the dashed lines in the images are shown in Figs. $2 \mathrm{f}$ and $2 \mathrm{~h}$. The dielectric EFM images clearly show the presence of the flagellum especially at the closest tip-substrate distances $(<40 \mathrm{~nm})$, with dielectric contrasts up to $10-20 \mathrm{zF} / \mathrm{nm}$ well above the instrumental noise of $\sim 0.7-2 \mathrm{zF} / \mathrm{nm}$. Note that the dielectric images display regions showing slightly different contrasts. The reason being that the flying tip-substrate distance is set at the ends of each line, and this may slightly change due to the presence of an unavoidable thin layer of residues $<1 \mathrm{~nm}$. However, this issue does not constitute any problem for the analysis, which is done line by line (see Materials and Methods).

To quantify the dielectric constant of the flagella we first calibrated the tip geometries, giving a tip radius $R_{s o}=23.9 \pm 0.5$ $\mathrm{nm}$, half cone angle $\vartheta_{S o}=11.5$ o and capacitance gradient offset offset $_{s_{0}}=118.5 \pm 0.7 \mathrm{zF} / \mathrm{nm}$ for the tip used in the $S$. oneidensis MR-1 flagellum measurements, and $R_{P a}=30.0 \pm 0.5 \mathrm{~nm}, \vartheta_{P a}=$ $11.5^{\circ}$, and offset $t_{P a}=128 \pm 2 \mathrm{zF} / \mathrm{nm}$ for the tip used in the $P$. aeruginosa PAO1 measurements (see Materials and Methods and Supplementary Information). The tip geometries, together with the deconvoluted flagella geometries reported above, are used to calculate numerically theoretical capacitance gradient images (see Materials and Methods section and Supplementary Information). Examples of calculated dielectric images at different tip-substrate distances and for different dielectric constants are shown in Figs. $3 \mathrm{a}$ and $3 \mathrm{~b}$, respectively, together with the corresponding capacitance gradient cross-section profiles shown in Fig. 3c and 3d. For numerical efficiency, we used a straight "short" $(I=1 \mu \mathrm{m})$ flagellum geometry, instead of the actual long-curved geometry displayed by the flagella (see Figs. $2 \mathrm{~b}$ and $2 \mathrm{c}$ ). This approximation is justified by the locality of the EFM measurements and the moderated curvature of the actual flagella (see Supplementary Information). Figures $3 e$ and 3f show the calculated capacitance gradient maxima as a function of the dielectric constant of the $S$. oneidensis MR-1 and $P$. aeruginosa $\mathrm{PAO} 1$ flagella, respectively, at four tip-substrate distances $\left(z_{\mathrm{CH}}\right)$ (continuous lines). The calculated curves are compared with the experimental measured capacitance gradient values at the center of the flagella (black symbols). The intercept gives the dielectric constant. We obtain dielectric constant values of $\varepsilon_{S o}=4.3 \pm 0.6$ and $\varepsilon_{P a}=4.5 \pm 0.7$ for $S$. oneidensis MR-1 and P. aeruginosa PAO1 flagella, respectively, where the error represents, here, the standard deviation of the values obtained at the four tip-substrate distances in each case. 

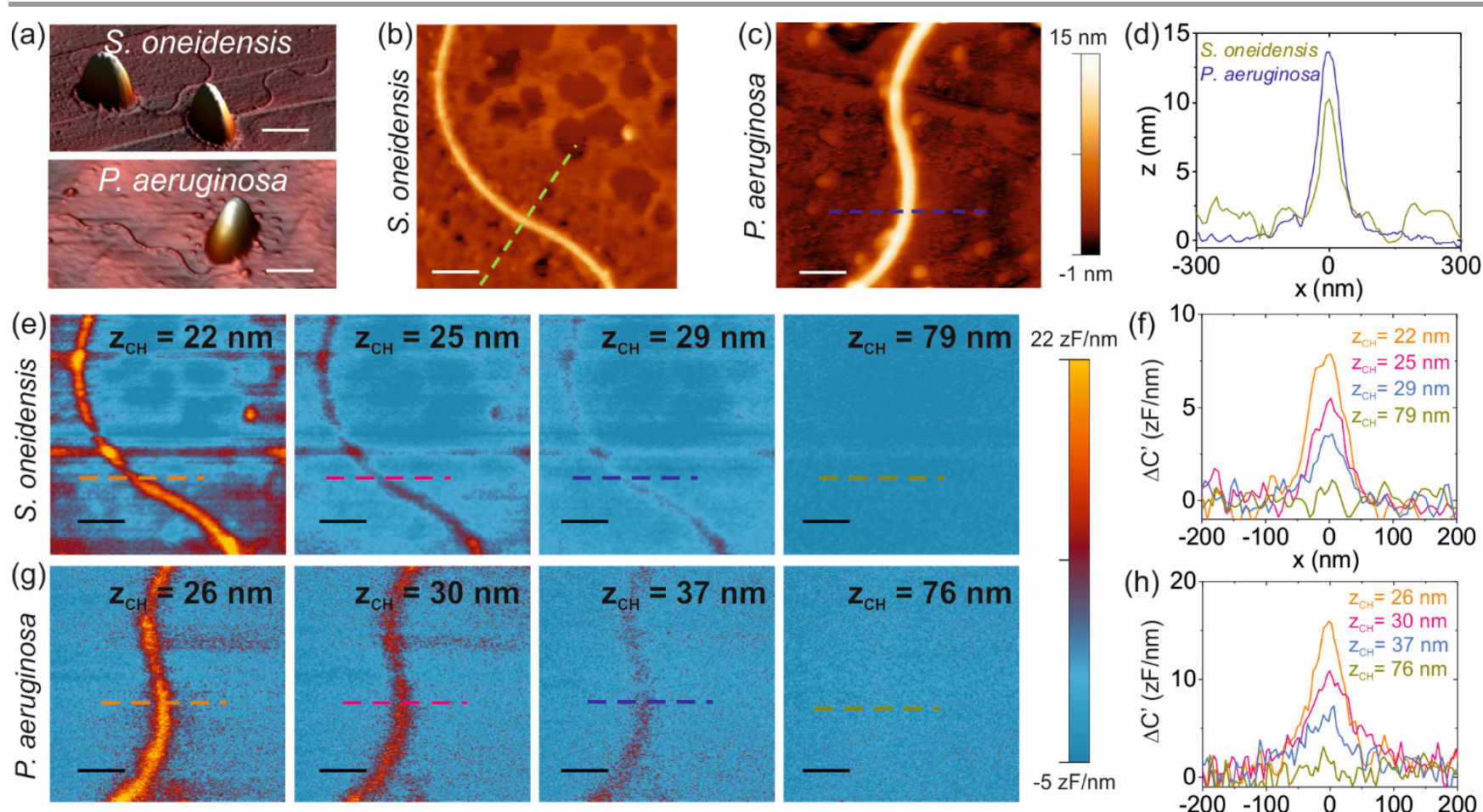
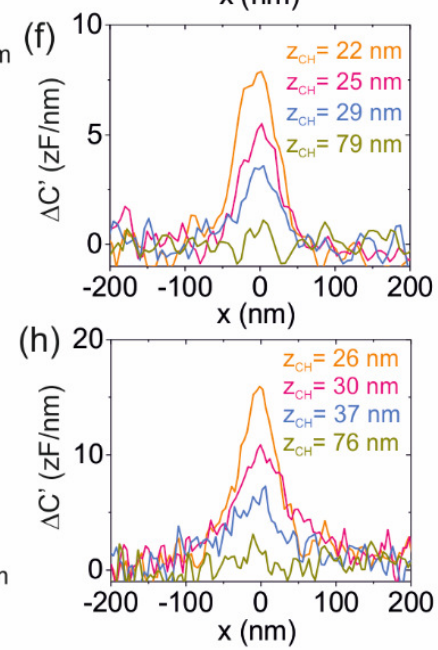

Figure 2: (a) Topographic AFM images of two S. oneidensis MR-1 and one P. aeruginosa PAO1 bacterial cells on an HOPG substrate. (b) and (c) zoom-ins of the images in (a) around the respective flagella. (d) Corresponding topographical cross-section profiles along the dashed lines in (b) and (c). (e) Capacitance gradient EFM contrast images of the S. oneidensis MR-1 flagellum at four different tip-substrate distances. (f) Corresponding capacitance gradient cross-section profiles along the dashed lines in (e). (g) and ( $\mathrm{h}$ ) idem for a flagellum from P. aeruginosa PA01. The scale bars in the images in (a) correspond to a length of $1.5 \mu \mathrm{m}$ and in (b) and (c) to $150 \mathrm{~nm}$. Parameters of the EFM measurements: applied voltage $V_{a c}=3 \mathrm{~V}$ and frequency $f=2 \mathrm{kHz}$, scan frequency $=1$ line $/ \mathrm{sec}$.

Figures $3 e$ and $3 f$ also show the capacitance gradient values measured on the bare HOPG substrate (grey symbols), which are used to set the tip-substrate distances (and hence matching the dielectric constant of unity by definition). The reliability of the extracted dielectric constant values has been further confirmed by comparing experimental capacitance gradient approach curves measured on top of the flagella with theoretically calculated ones, and by comparing the full experimental capacitance gradient EFM profiles with the calculated ones (see Supplementary Information). Remarkably, after considering the respective tip and sample geometries, the dielectric constant values obtained for the two types of flagella are almost the same. The same procedure described above was repeated for three different additional flagella of $S$. oneidensis, obtaining in all cases similar values for the extracted dielectric constant (see Fig. 3g). The overall ensemble average value for the relative dielectric constant $(N=4)$ is $\varepsilon_{S_{o}}=4.1 \pm 0.4$ (see Supplementary Information for additional data).

Determining the dielectric constant of proteins is an inherently complex problem, due to its microscopic nature and to its unavoidable interaction with the solvent or environment (e.g. solution electrolytes or the cell membrane). In fact, measurements performed on protein solutions (e.g. by impedance spectroscopy), reflect both the dielectric properties of the proteins, as well as those of the protein-solvent interface (21) and interfacial water molecules (22). On the other side, measurements performed on dry crystal powders reflect only partially the structure and dynamics of proteins in native conditions (6). Scanning Dielectric Microscopy enables measuring the dielectric constant in dry conditions, to get rid of solution effects, and on natural protein macromolecular complexes.

In this work, we measured the dielectric constant of the filamentous part of polar flagella, composed of flagellin proteins (23), of two distinct types of bacteria, namely S. oneidensis MR1 and $P$. aeruginosa PAO1. The obtained dielectric constants fall within a relatively short range of values $\varepsilon_{r} \sim 4-4.5$, well defined by the small uncertainty of the measurements (less than a $\sim 15 \%$, despite the complexity of the measurements and the small size of the flagella). We note that while the amino acid sequences of the terminal regions of flagellin, including about $180 \mathrm{NH}_{2}$-terminal and $100 \mathrm{COOH}$-terminal residues, are known to be well conserved from species to species of bacteria, the central region can be highly variable (24). In the case of $P$. aeruginosa flagellin protein (FliC) is 122 aminoacids longer at the central region of the protein compared to the $S$. oneidensis flagellin. Despite that, the dielectric response of flagella from $P$. aeruginosa $\mathrm{PAO} 1$ and $\mathrm{S}$. oneidensis MR- 1 was very similar. 
(a)

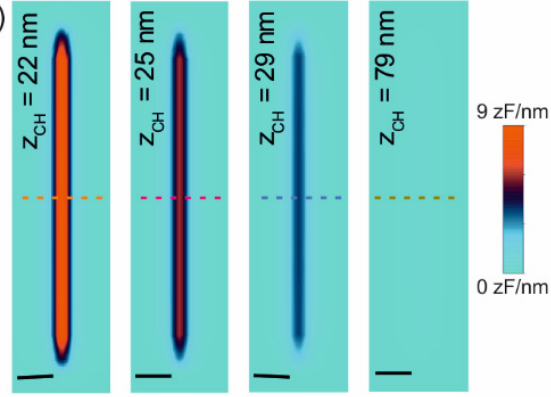

(e)

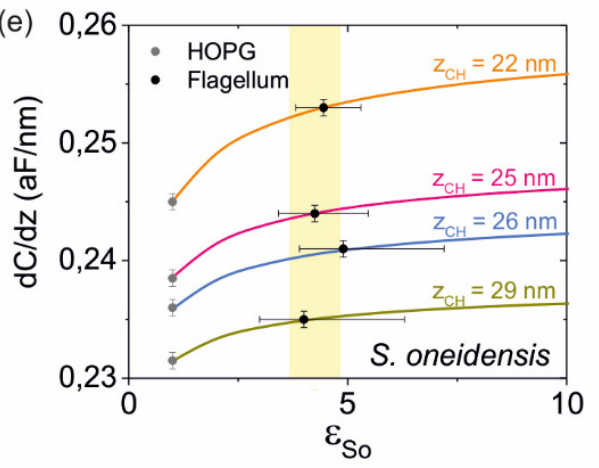

(b)

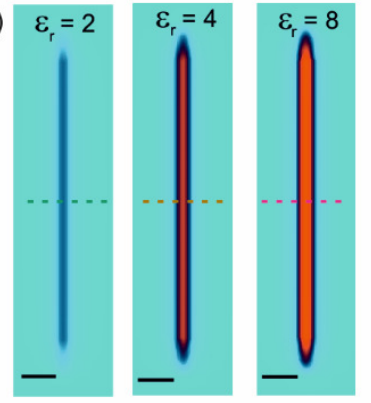

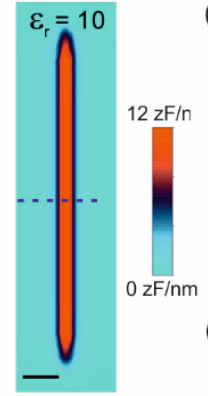

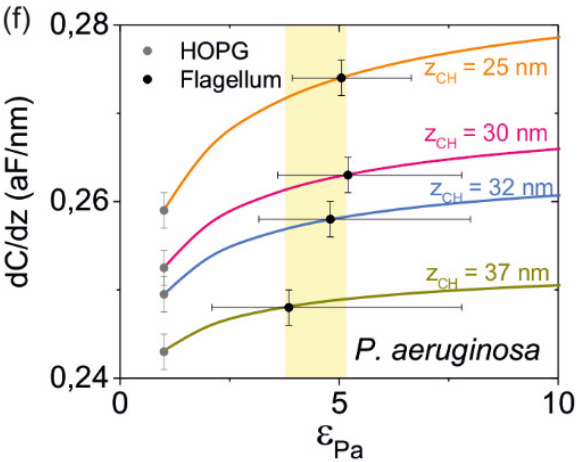

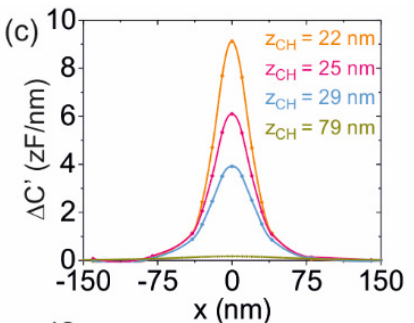

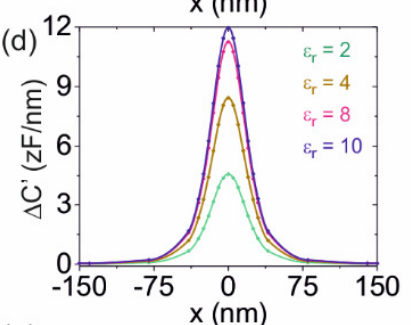

(g)

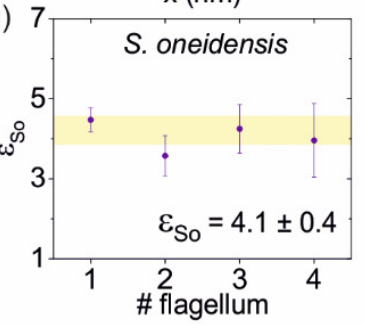

Figure 3: (a) Examples of calculated capacitance gradient contrast EFM images at four different distances $z_{C H}=22,25,29$ and $79 \mathrm{~nm}$ of a straight flagellum with given dielectric constant $\varepsilon_{r}=4.3$. The tip and flagellum geometry correspond to the ones used to quantify the $S$. oneidensis MR-1 flagellum (see below). (b) Idem but for a given tip substrate distance $z_{C H}=$ $22 \mathrm{~nm}$ and four different dielectric constants of the flagellum, $\varepsilon_{r}=2,4,8,10$. (c) and (d) Corresponding capacitance gradient cross-section profiles along the dashed lines shown in (a) and (b). (e) Calculated capacitance gradient profile maxima as a function of the dielectric constant of the $S$. oneidensis MR-1 flagellum for four tip-substrate distances (continuous

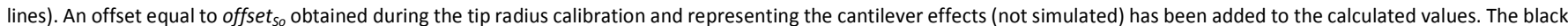
and grey symbols represent, respectively, the experimental capacitance gradient values obtained from the EFM images in Fig. 2 at the center of the flagellum and on a bare part of the substrate. The later ones are used to set the tip sample distance and hence they are set to $\varepsilon_{r}=1$. The average dielectric constant value is $\varepsilon_{S_{0}}=4.3 \pm 0.6$ (shadowed area). (f) Idem for the case of the $P$. aeruginosa PA01 flagellum, giving $\varepsilon_{P a}=4.5 \pm 0.7$ (shadowed area). (g) Dielectric constant values measured on four different $S$. oneidensis flagella. The overall average dielectric constant value is $\varepsilon_{S_{0}}=4.1 \pm 0.4$ (shadowed area). Parameters used in the $S$. oneidensis calculations: $R_{S o}=23.9 \mathrm{~nm}, \vartheta_{S o}=11.50, h_{S o}=8.4 \mathrm{~nm}, w_{S_{o}}=18 \mathrm{~nm}$ and $l_{S_{0}}=$ $1000 \mathrm{~nm}$, offset $t_{S o}=118.5 \mathrm{zF} / \mathrm{nm}$. Parameters used in the $P$. aeruginosa calculations: $R_{P a}=30.0 \mathrm{~nm}, \vartheta_{P a}=11.5 \circ, h_{P a}=13.0 \mathrm{~nm}, w_{P a}=27 \mathrm{~nm}$ and $l_{P a}=1000 \mathrm{~nm}$, offset $t_{P a}=128.5 \mathrm{zF} / \mathrm{nm}$. For the specific parameters used in $(\mathrm{g})$ see Supplementary Information.

Furthermore, the dielectric constant found for the flagella is is similar to the values obtained by means of the same technique in other macromolecular protein complexes, such as the T7 bacteriophage virus capsid $\left(\varepsilon_{r}=3.5 \pm 0.5\right)(8)$ and virus tail $\left(\varepsilon_{r}=\right.$ $3.4 \pm 0.4)$ (9), or submicrometric monolayer patches of bacteriorhodopsin $\left(\varepsilon_{r}=3.3 \pm 0.3\right)(10)$. However, they differ from those obtained on macromolecular complexes of other cellular components, such as micrometric lipid bilayers patches (DOPC, $\left.\varepsilon_{r}=1.9 \pm 0.3\right)$, cholesterol crystals $\left(\varepsilon_{r}=2.1 \pm 0.3\right)$ ) (10), T7 bacteriphage full viruses $\left(\varepsilon_{r}=6.3 \pm 0.4\right)$ (8) and T7 bacteriophage DNA $\left(\varepsilon_{r}=8.5 \pm 1.4\right)$ (9). Present results thus support the use of dielectric constant values in the range $\varepsilon_{r}$ 3-4 for the hydrophobic part of proteins, which is believed to account for electronic polarization and small backbone fluctuations (4). Whether there is a functional reason for the dielectric constant of proteins to take values in the intermediate range between that of lipids and DNA, or whether there are some proteins showing markedly different dielectric behaviour, still needs to be further investigated.

The SDM dielectric measurements reported here are local and at the nanoscale. The dielectric constant obtained for the flagella reflects the dielectric constant of a very small portion of the flagella. The length of this portion, referred to as dielectric probed length, can be determined as the length of the flagellum starting from which the dielectric contrast reaches a value independent from the length of the flagellum (see Supplementary Information). Figure 4 (black symbols, left axis) shows the dependence of the dielectric probed length as a function of the tip radius for case of the $S$. oneidensis flagellum. For the tip radius used in the present study $(\sim 25 \mathrm{~nm})$, the probed length of the flagella is $\sim 130 \mathrm{~nm}$, which corresponds to $\sim 250$ flagellin protein monomers (17), which is a remarkably small number.

Improving the locality of the dielectric measurement on full flagella structures, and hence probing a smaller number of protein monomers, is barely possible by using the presented approach, since further reducing the tip radius and/or the cone angle only moderately improves the locality of the dielectric measurement (see the tendency of the dielectric probed length in Fig. 4 for the smallest radii). Moving to force gradient detection could improve somehow the locality of the measurement (the dielectric probed length in this case reduces by a factor 0.5-0.7, see Supporting Information), but still the number of proteins probed is relatively large. Probing a significantly smaller number of flagellin protein monomers using this technique would require considering flagella portions 
(instead of full flagella), with a length shorter than the dielectric probed length discussed above. Under these conditions and based on previous studies (10), it should be possible to attain the few flagellin monomer limit.

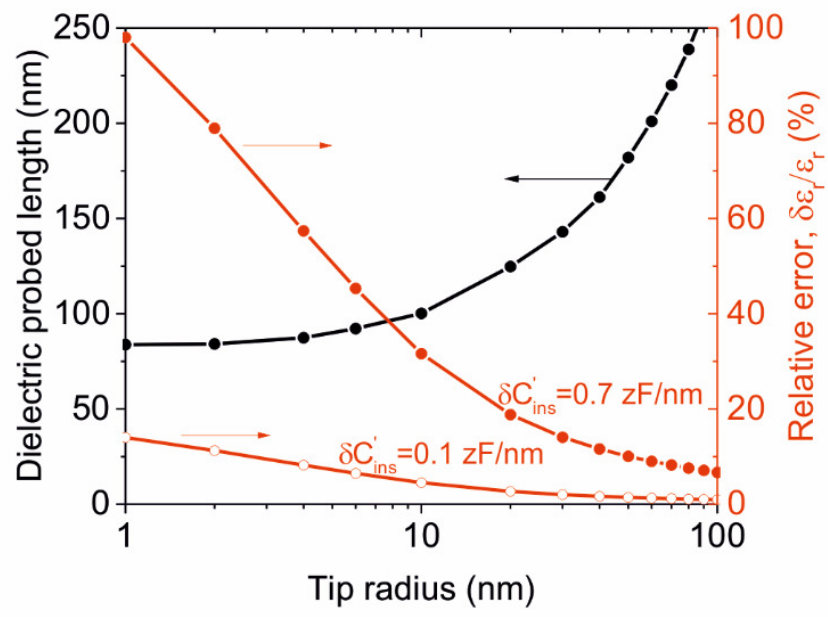

Figure 4: (Left axis, black symbols) Numerical calculated length of the flagellum probed dielectrically by means of the SDM measurements as a function of the tip radius. (Right axis, red symbols) Relative error in the extracted relative dielectric constant as a function of the tip radius for two different instrumental noise levels $\delta C^{\prime}{ }^{\prime}$ int $=0.7 \mathrm{zF} / \mathrm{nm}$ (filled red symbols) and $0.1 \mathrm{zF} / \mathrm{nm}$ (empty red symbols). Lines are guides to the eyes. Parameters used in the calculations: $R=23.9 \mathrm{~nm}, \vartheta=11.5$ o,$h=$ $8.4 \mathrm{~nm}, w=18 \mathrm{~nm}, l=1000 \mathrm{~nm}, \varepsilon_{r}=4.1$ and $z_{C H}=22 \mathrm{~nm}$.

Reducing the tip radius, or the length of the flagella would increase the uncertainty of the dielectric constant due to a reduction of the signal to noise ratio. We show it in Fig. 4 (red symbols, right axis) where we plot the relative error in the dielectric constant extraction as a function of tip radius for the instrumental noise level of the present work, $\delta C^{\prime}{ }_{i n s t}=0.7 \mathrm{zF} / \mathrm{nm}$ and for the state of the art noise level, $\delta C^{\prime}{ }_{\text {inst }}=0.1 \mathrm{zF} / \mathrm{nm}(8)$. The relative error has been calculated according to the relation

$$
\frac{\delta \varepsilon_{r}}{\varepsilon_{r}}=\frac{\delta C_{\text {inst }}^{\prime}}{\varepsilon_{r} \frac{\partial}{\partial \varepsilon_{r}} \Delta C^{\prime}\left(z, \varepsilon_{r}, R\right)}
$$

where $\Delta C^{\prime}(z, \varepsilon, R)$ is the maximum contrast for a given tipsubstrate distance, $z$, relative dielectric constant of the flagellum, $\varepsilon_{r}$, and tip radius, $R$ (see Supplementary Information). For the tip radii used in the present work $(\sim 25 \mathrm{~nm})$ the relative error in a single measurement is $\sim 15 \%$, which can be reduced below a $\sim 10 \%$ by averaging several measurements. However, for smaller tip radii $(\sim 5 \mathrm{~nm})$, or for short portions of flagella, the error in the extraction of the dielectric constant would quickly rise to unacceptable values $(>30 \%)$, unless the instrumental noise is decreased down to at least $\sim 0.1 \mathrm{zF} / \mathrm{nm}$ (empty red symbols in Fig. 4).

On wet samples the overall dielectric response of proteins might slightly increase due to contributions coming from polar end groups and from eventual tightly adsorbed water molecules. The contribution of the hydrophobic core of the protein, instead, is expected not to be significantly affected. To this respect we note that recent findings on the very low dielectric constant of confined water (22) might give rise to a smaller increase in the dielectric response of the shell region than expected.

Finally, we remark that the present approach should be applicable to most filamentous protein nanostructures present in cells, such as actin or myosin filaments, microtubules or intermediate filaments, including proteins nanofilaments relevant for some diseases, such as amyloid fibers (17), thus spanning significantly the number of proteins accessible to dielectric quantification.

\section{Materials and Methods}

Scanning Dielectric Microscopy. We obtained dielectric images by using EFM (19), (20) in amplitude detection mode at constant height, as described in Ref (8). Data are reported in terms of the capacitance gradient, which is related to the electrostatic force at the $2 \omega$ harmonic, $F_{2 \omega}$, by $C^{\prime}(z)=4 F_{2 \omega}(z) / V_{a c}{ }^{2}$, where $V_{a c}$ is the amplitude of the ac applied voltage. Capacitance gradient approach curves were measured on the substrate to determine the tip-substrate distance at which each line has been acquired, and, to calibrate the tip geometry, following the tip calibration procedure explained elsewhere (8) (see also Supplementary Information).

Measurements where performed with a commercial AFM (25), Cypher $\mathrm{S}$ and its internal lock-ins from Asylum Research, using its internal lock-in to apply the ac voltage and to read the amplitude and phase of the electrostatic probe oscillation. Measurements were performed in controlled dry air conditions $(\mathrm{RH}<1 \%)$ maintained by a $\mathrm{N}_{2}$ flow. We used platinum silicide conductive probes (PtSi-CONT, Nanosensors) with a spring constant $k \sim 0.2 \mathrm{~N} / \mathrm{m}$ (determined by the provider according to the probe dimensions), resonance frequency $f_{r} \sim 13 \mathrm{kHz}$, nominal radius $R \sim 20 \mathrm{~nm}$ and half cone angle $\vartheta \sim 11.5$. The analysis of the data was done using the WSxM software (26) (Nanotec Electrónica S.L.) and a custom-made software developed in Matlab (The Mathworks, Inc). Dielectric images were obtained in the SNAP mode of the Cypher S (the built-in line by line constant height mode). All EFM data were obtained with a voltage amplitude $V_{a c}=3 \mathrm{~V}$ at frequency $f=2 \mathrm{kHz}$ (well below the mechanical resonance peak). The capacitance gradient instrumental noise was in the range $\sim 0.7-2 \mathrm{zF} / \mathrm{nm}$ depending on the probe used and recording parameters.

Finite-Element Numerical Calculations. We used finite element numerical calculations to simulate theoretical EFM capacitance gradient images and approach curves. These data were used in the quantitative analysis of the experimental EFM measurements. The tip was represented like in previous works (27), (28) as a truncated cone of half-angle $\theta$ and cone height $\mathrm{H}$ terminating in a tangent hemisphere of radius R. A disc of thickness $W c$ and with a radius that oversees the cone base by an amount Lc is located onto the cone base, and it models local cantilever effects. Nonlocal cantilever contributions have been considered through a phenomenological capacitance gradient offset contribution term. We used a single angle cone model, as in Ref. (8), instead of a two-angle cone model that would better reflect the real geometry of the PtSi-CONT tips, since measurements are restricted to very short distances $(<70 \mathrm{~nm})$. 
In this distance range single and double cone angle models provide almost identical results (28), while single angle models largely facilitate its 3D modelling.

The flagellum is modeled as a straight elliptic cylinder with axes $\mathrm{w}$ and $\mathrm{h}$ for the base ellipse, and length $\mathrm{I}$. The flagellum is assumed to lie on a metallic substrate. Non-straight flagella geometries (e.g., curved elliptic cylinder) have also been considered, producing negligible differences with respect to a straight elliptic cylinder for the actual experimental geometries (see Supplementary Information). The straight geometry has been kept for computational efficiency. A schematic threedimensional representation of the tip-flagellum model used for the numerical calculations is shown in the Supplementary Information.

The capacitance gradient between the tip and the sample was calculated by solving Poisson's equation with the AC/DC electrostatic module of Comsol Multiphysics 5.3. We set the surface of the tip to "terminal", the bottom boundary of the simulation domain to "ground", and the top and side boundaries to "zero charge". The infinite element function is used on the top and side boundaries to get rid of finite size effects of the simulation domain. We calculated the electric force on the tip by integration of the Maxwell stress tensor on the tip surface (see further details in Ref. (8)). The simulation domain was cylindrical with radius $\sim 16 \mu \mathrm{m}-25 \mu \mathrm{m}$ and a height $\sim 30 \mu \mathrm{m}-50 \mu \mathrm{m}$, depending on tip position with respect to the filament. The mesh was set to a minimum of $6 \cdot 10^{5}$ elements (see Supplementary Information). An accurate process of optimization, validation, and numerical noise reduction of the 3D simulations has been undertaken, to meet sub-0.1 zF/nm noise accuracy (see Supporting Information).

Automatic software routines written in Matlab (The MathWorks, Inc.) have been used to compute capacitance gradient images. In the calculations, the tip moves at constant height with respect to the substrate. In addition, capacitance gradient approach curves have been also calculated by varying the tip-substrate distance.

Probe geometry calibration. The tip geometry has been determined as in Ref. (8) by fitting a short range (15-60 nm) capacitance gradient approach curve measured over a bare part of the metallic substrate with calculated theoretical capacitance gradient approach curves, with the tip radius and capacitance gradient offset as free parameters. The remaining tip parameters were set to their nominal values: $\theta=11.5$ ㅇ $H=12.5$ $\mu \mathrm{m}, L_{c}=3 \mu \mathrm{m}$ and $W_{c}=3 \mu \mathrm{m}$. As we mentioned above, the use of single cone angle model tip is justified for the sharpened tips used in the present work because only short-range distances are considered in the EFM approach curves used for the tip calibration and in the image acquisition (29).

Flagellum width deconvolution. The flagella width, w, was obtained by fitting the measured topographic profile with the analytical spherical tip-ellipse convolution expression,

$$
Z_{\text {conv }}(X)=\left(\frac{h}{2}-\frac{w^{2}}{2 h}\right) \sin (\alpha(X))+X \frac{w}{h} \tan (\alpha(X))-R+\frac{h}{2}
$$

$$
X=\frac{w}{2} \cos (\alpha(X))+\frac{R}{\sqrt{1+\left(\frac{w}{h} \tan (\alpha(X))\right)^{2}}}
$$

where $h$ is the measured flagella height and $R$ is the calibrated tip radius. Equations ( 2 ) and ( 3 ) can be used to calculate the tip convoluted profiles if the tangent point between the tip apex and the ellipse is at a height smaller than $R(1-\sin (\theta))$, which was the case for the tips used in the present work. For smaller tip radii one must introduce also the contribution of the tip cone in the calculation of the tip convolution. A written Matlab (The MathWorks, Inc.) code was written to implement this procedure automatically. Further details are given in the Supplementary Information.

Dielectric constant extraction. The relative dielectric constant of the flagella $\varepsilon_{\text {flag }}$ was determined as in Ref. (8) from the experimental capacitance gradient images obtained at different tip-substrate distances, by considering the values of the capacitance gradient contrast at the center of the flagella (maximum). These experimental capacitance gradient values were compared with simulated capacitance gradient curves as a function of the flagellum dielectric constant numerically obtained by using the deconvoluted flagellum geometry and the calibrated tip geometry at the given experimental tip sample distance. The extracted values for $\varepsilon_{\text {flag }}$ from a given image correspond to the mean and standard deviation of the extracted values for the different tip-substrate distances.

Bacteria growth and sample preparation. We used Shewanella oneidensis MR-1 (ATCC 700550) cultivated first overnight at 30 ㄷ $\mathrm{C}$ in Luria-Bertani (LB) (Scharlab) broth in aerobic conditions, and then in minimal $A B$ medium (30) supplemented with 100 $\mathrm{mM}$ fumarate and $20 \mathrm{mM}$ lactate during two days in anaerobic conditions at $30{ }^{\circ} \mathrm{C}$ in Hungate tubes. We also used Pseudomonas aeruginosa PAO1 (ATCC 15692) grown overnight in LB medium at 37 ㅇ $\mathrm{C}$ in aerobic conditions. The samples for EFM inspection are prepared by taking a drop of the culture solutions, depositing it on a freshly cleaved HOPG and leaving it to rest for $10 \mathrm{~min}$. Afterwards, the drop sample is rinsed two times, first with $\mathrm{PBS}$ and second with mili-Q water to remove any poorly adhered cells or any residual from the sample. Finally, we left the sample to dry in ambient conditions. The HOPG substrate was attached to a $1.5 \mathrm{~cm}$ diameter magnet using a carbon double side stick, which was connected to the electrical ground of the atomic force microscope by a small wire.

\section{Conclusions}

We have used Scanning Dielectric Microscopy to locally measure the dielectric constant of the filamentous part of bacterial polar flagella composed of flagellin proteins. We have obtained dielectric constant values $\varepsilon_{S o}=4.3 \pm 0.6$ and $\varepsilon_{P a}=4.5$ \pm 0.7 , for flagella belonging to two distinct types of bacteria, namely, S. oneidensis MR-1 and $P$. aeruginosa PAO1, respectively. These values do not depend on the bacterial species and reflect the response of only a few hundred proteins. Similar relative dielectric constant values have been reported 
for other macromolecular protein complexes using the same technique. Altogether, these results indicate that the relative dielectric constant of proteins in dry conditions show dielectric constant values ( 3-5), like those reported using macroscopic techniques applied to dry protein crystals, and different from the values reported for other cell components such as lipids ( 2) or DNA ( 8). These results may have important implications in the understanding of the relevance of the electrostatic energy contribution to protein function and structure.

\section{Conflicts of interest}

There are no conflicts to declare.

\section{Acknowledgements}

This work has been partially supported by the Spanish Agencia Estatal de Investigación and EU FEDER through grants TEC201572751-EXP, TEC2016-79156-P and BIO2015-63557-P. G. G. acknowledges support from an ICREA Academia award from the Generalitat de Catalunya and H. L. for an FPI grant (BES-2015074799) from the Agencia Estatal de Investigación/Fondo Social Europeo. We also acknowledge support from Generalitat de Catalunya (2017 SGR1079 and CERCA Program).

\section{References}

1 B. Honig, A. Nicholls, Science 1995, 268, 1144.

2 K. A. Sharp, B. Honig, Annu. Rev. Biophys. Biophys. Chem. 1990, 19, 301.

3 M. K. Gilson, B. H. Honig, Biopolymers, 1986, 25, 2097.

4 T. Simonson, C. L. Brooks, J. Am. Chem. Soc., 1996, 118, 8452.

5 C. N. Schutz, A. Warshel, Proteins, 2001, 44, 400.

6 T. Simonson, Rep. Prog. Phys., 2003, 66, 737.

7 M. F. Perutz, Science, 1978, 201, 1187.

8 L. Fumagalli, D. Esteban-Ferrer, A. Cuervo, A. J. L. Carrascosa, G. Gomila, Nat. Mat., 2012, 11, 808.

9 A. Cuervo, P. D. Dans, J. L. Carrascosa, M. Orozco, G. Gomila, L. Fumagalli, Proc. Natl. Acad. Sci., 2014, 111, E3624.

10 A. Dols-Perez, G. Gramse, A. Calo, G. Gomila, L. Fumagalli, Nanoscale, 2015, 7, 18327.

11 S. T. Bayley, Trans. Faraday Soc., 1951, 49, 509.

12 S. Takashima, H. P. Schwan, J. Phys. Chem., 1965, 69, 4176.

13 M. Bockrath, N. Markovic, A. Shepard, M. Tinkham, L. Gurevich, L. P. Kouwenhoven, Nano Lett., 2002, 2, 187.

14 W. Lu, D. Wang, L. Chen, Nano Lett., 2007, 7, 2729.

15 W. Lu, Y. Xiong, A. Hassanien, W. Zhao, Mi. Zheng, L. Chen, Nano Lett., 2009, 9, 1668.

16 Y. Yang, W. Guo, X. Wang, Z. Wang, J. Qi, Y. Zhang, Nano Lett. 2012, 12, 1919.

17 T. P. J. Knowles, M. Vendruscolo, Ch. M. Dobson, Nat. Rev. Mol. Cell Biol., 2014, 15, 384.

18 F. Wang, A. M. Burrage, S. Postel, R. E. Clark, A. Orlova, E. J. Sundberg, D. B. Kearns, E. H. Egelman, Nat. Commun. 2017, 8, 960.

19 Y. Martin, D. W. Abraham, H. K. Wickramasinghe, Appl. Phys. Lett. 1988, 52, 1103-1105.

20 P. Girard, Nanotechnology 2001, 12, 485-490.

21 R. Pethig, Ann. Rev. Phys. Chem., 1992, 43, 177.

22 L. Fumagalli, A. Esfandiar, R. Fabregas, S. Hu, P. Ares, A. Janardanan, Q. Yang, B. Radha, T. Taniguchi, K. Watanabe, G. Gomila, K. S. Novoselov, A. Geim, Science, 2018, 360, 1339
23 T. Fujii, T. Kato, K. D. Hiraoka, T. Miyata, T. Minamino, F. F. V. Chevance, K. T. Hughes, K. Namba, Nat. Commun. 2017, 8, 14276.

24 D. R. Wilson, T. J. Beveridge, Can. J. Microbiol., 1994, 39, 67.

25 P. Eaton, P. West, Atomic Force Microscopy (Oxford University Press, Oxford, 2010).

26 I. Horcas, R. Fernandez, J. M. Gomez-Rodriguez, J. Colchero, J. Gomez-Herrero, Rev. Sci. Instrum., 2007, 78, 013705.

27 M. C. Biagi, R. Fabregas, G. Gramse, M. Van Der Hofstadt, A. Juarez, F. Kienberger, L. Fumagalli, G. Gomila, ACS Nano, 2016, $10,280$.

28 M. Van Der Hofstadt, R. Fabregas, R. Millan-Solsona, A. Juarez, L. Fumagalli, G. Gomila, ACS Nano, 2016, 10, 11327.

29 L. Fumagalli, M. A. Edwards, G. Gomila, Nanotechnology, 2014, 25, 495701.

30 N. Uría, X. Muñoz Berbel, O. Sánchez, F. X. Muñoz, J. Mas, Environ. Sci. Technol. 2011, 45, 10250. 